\title{
Manejo y gestión de residuos sólidos urbanos en la región de Cataluña (NE de España), legislación y educación ambiental
}

\author{
Emma Gema García González ${ }^{\mathrm{a} *}$, Juan Alvárez Cavazos ${ }^{\mathrm{a}}$ \\ ${ }^{a}$ Universidad Autónoma de Nuevo León, UANL, FCT, Ex-Hacienda de Guadalupe Carr. Linares-Cerro Prieto km. 8, Linares, N.L. \\ *emma.garciagn@uanl.edu.mx
}

Recibido 23 de febrero 2015, Aceptado 30 junio 2015

\section{Resumen}

Se ha llevado a cabo un análisis en relación a los residuos sólidos urbanos, tomando como gestión de los mismos el plan piloto en la región de Cataluña (España). Abarca tres etapas: Depósito y recogida, transporte y tratamiento; siendo de destacar por su protección al medio ambiente, el sistema neumático (fijo, móvil) con contenedores soterrados, además de los centros de tratamiento constituidos por los puntos limpios o ecoparques. Por otro lado, la Comunidad Autónoma de Cataluña dispone de competencias en materia de residuos, y por tanto, se presenta la legislación aplicable en todo el territorio autonómico. En relación a la educación ambiental, se están impulsando programas educativos en algunos municipios, la "Red de Escuelas Verdes", construcción y dinamización de centros especializados como son: Centros de Educación Ambiental, Centros de Interpretación de la Naturaleza, Casas de Naturaleza, o Centros de Divulgación de la Gestión de la Basura. Finalmente, en el área metropolitana de Barcelona se ha establecido el Consejo de Seguimiento del Programa Metropolitano de Gestión de Residuos Municipales con el fin de dar seguimiento al Programa de Residuos.

Palabras clave: Residuos sólidos, gestión, legislación, educación ambiental.

\begin{abstract}
An analysis of solid municipal waste was conducted, modeled on the Cataluña pilot project. It covers three stages: Waster collection, transport and treatment - centered on environment protection - ; the pneumatic system (fixed, mobile), with underground containers; and treatment centers consisting of clean points or eco-parks. However, as the Cataluña Autonomous Region (Spain) has experience in waste disposal, the appropriate legislation applies throughout its territory. Concerning environmental education, educational programs such as the "Red de Escuelas Verdes" ("Web of Green Schools") are being implemented in certain cities, as well as the construction and enhancement of specialized centers. Examples of the latter include: The Environmental Education Center, the Nature Interpretation Center, Nature Homes, and the Waste Management Outreach Center. Finally, in order to better monitor its waste management program, the city of Barcelona established the Metropolitan Municipal Waste Management Council.
\end{abstract}

Key words: Solid waste, management, legislation, environmental education.

\section{Introducción}

Las ciudades y las personas tenemos ante nosotros el importante deber de velar por la sostenibilidad, la protección del medioambiente, los recursos y el bienestar de la sociedad.

Se han dedicado grandes esfuerzos humanos y económicos con el fin de fomentar el intercambio de conocimiento, tecnología, experiencias y formación.

La Universidad Politécnica de Cataluña intenta aportar los valores técnicos de la gestión eficiente de los residuos municipales, defender los valores de la educación ambiental, participación ciudadana en este proceso, respeto a las tecnologías limpias y adaptadas a las realidades y necesidades de los distintos países, en armonía con las culturas y lenguas propias.

La actividad humana basada en la explotación de recursos naturales ha crecido en grandes cantidades en los últimos años proporcionando una considerable mejora en las condiciones de vida. Fruto de esta actividad es la producción creciente de residuos, suponiendo un problema en el proceso de eliminación.

El crecimiento demográfico y el consumo exagerado debido al aumento del nivel de vida son las principales causas de la producción de residuos en grandes masas.
Las soluciones, no pueden dirigirse únicamente a la reducción de ese consumo, sino que han de dirigirse hacia la reutilización y recuperación de los residuos.

El presente trabajo, tiene como objetivo compartir la experiencia del programa de gestión de los residuos sólidos urbanos en la provincia de Cataluña, como un modelo a seguir, teniendo en cuenta los planteamientos y posibilidades de realización para ser extendido a otras ciudades del mundo, dando prioridad a la separación de la materia orgánica para posteriormente sufrir una transformación en compost de alta calidad e integración en el proceso de compostaje en la agricultura. Asimismo, concientizar a la población de la recogida selectiva en origen de, al menos, las fracciones siguientes: Materia orgánica fermentable, residuos correspondientes a vidrio, papel, cartón, residuos peligrosos y voluminosos. Respecto a la educación ambiental, se debe planificar la introducción de los conceptos de prevención, reutilización y reciclaje llevándose a cabo experiencias previas y voluntarias, teniendo un contenido experimental y práctico. Finalmente, elaborar una metodología que permita un seguimiento de Gestión Integral, teniendo en cuenta la preservación de la salud 
humana, mejora de la calidad de vida, cuidado del medio ambiente y conservación de los recursos naturales; para conseguir lo anteriormente citado, se debe implicar a toda la sociedad, mediante una participación activa de la ciudadanía que debe estar basada en la sensibilización, intervención política, gestión administrativa, y diversas instituciones. Es necesario modificar ciertos aspectos como aprendizaje, motivación, cambio de hábitos, etc, que son imprescindibles para fomentar el camino hacia la sostenibilidad.

\section{Parte experimental}

\subsection{Ubicación, definiciones y clasificación}

Cataluña, se encuentra situada al noreste de España, tiene una superficie aproximada de $32.000 \mathrm{~km}^{2}$ y una población de más de 13.712.983 de habitantes (Figura 1).

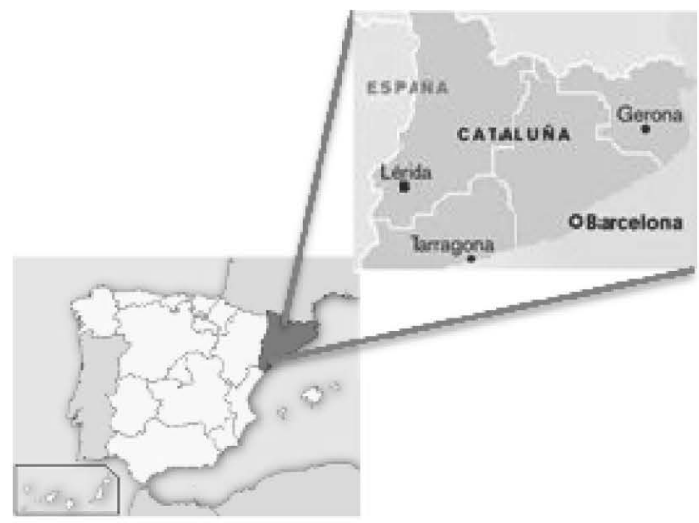

Figura 1. Localización de las provincias que comprende comunidad autónoma de Cataluña.

El concepto de residuo está definido de diversas formas:

Según la O.C.D.E. (Organización de Cooperación y Desarrollo Económico) se define el residuo como "aquellas materias generadas en las actividades de producción y consumo que no tienen, en el contexto en el que son producidas, ningún valor económico".

Como definición de Residuos Sólidos Urbanos, se entiende "a los generados en los domicilios particulares, comercios, oficinas y servicios, así como a todos aquellos que no tengan la calificación de peligrosos y que por su naturaleza o composición puedan asimilarse a los producidos en dichos lugares o actividades".

Tendrán también la consideración de residuos urbanos los siguientes: "los residuos procedentes de la limpieza de vías públicas, zonas verdes, áreas recreativas, playas, animales domésticos muertos, así como muebles, utensilios y vehículos abandonados, residuos de escombros procedentes de obras menores de construcción y reparación domiciliaria”.

En la figura 2 se presentan los datos en $\mathrm{kg} /$ persona de los residuos municipales generados en la Unión Europea, en el año 2009, donde España ocupa el $5^{\circ}$ hugar.

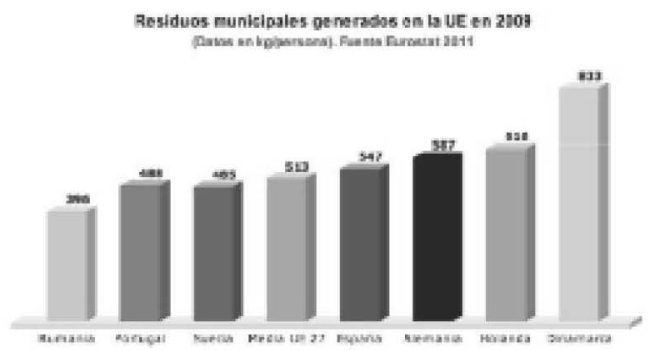

Figura 2. Datos de la oficina estadística de la Unión Europea, Eurostat.

En la Tabla 1 se representan los datos correspondientes a los residuos sólidos urbanos generados durante un estudio realizado el año 2010 en diferentes provincias españolas, destacándose que la provincia de Barcelona ocupa el segundo lugar de recogida de residuos sólidos urbanos, alcanzando el primer lugar la provincia de Madrid.

Tabla 1. Residuos sólidos urbanos generados el año 2010 en diferentes provincias españolas.

\begin{tabular}{|c|c|c|c|c|c|}
\hline \multicolumn{6}{|c|}{ AVO 2010} \\
\hline Clevintis & 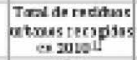 & 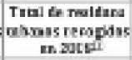 & Fretuden & 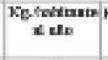 & $\begin{array}{l}\text { Kanowane } \\
\text { si bs }\end{array}$ \\
\hline ACanfas & :12mi & $1100)$ & an & $\Leftrightarrow$ & $1 . x$ \\
\hline Allaren & 249.58 & 1610.2 & $-16 \leqslant 8$ & 44 & 158 \\
\hline MTrFims & 142.120 & 2x:65 & $240 \%$ & 31 & $1,4:$ \\
\hline Hitas & 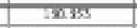 & $7 \pi 29$ & whos & $\Leftrightarrow$ & 72 \\
\hline Ditiots & tere treted & 17105 & Dab rs Equanv & Tmm & arwing \\
\hline Socuadu & $n \pi$ & 12244 & $12 \%$ & $5 \pi$ & 1.2. \\
\hline Laproua & $x \times 3$ & 1261 & $16 \%$ & mi & $|0|$ \\
\hline Marrs: & -472461 & $1 \% 01 \mathrm{a}$ & $1 \%$ & as & 1.2 \\
\hline walag & 314.31 & 30.39 & $1 \pi$ & wi & $1 \mathrm{H}$ \\
\hline Mharis & $2 k . \infty$ & Lados faritadx & Loda za ciquentak & se: & $12 i$ \\
\hline Salieb & 3114 & $45:-$ & $.1 \%$ & 나: & 1,11 \\
\hline Funikss & कार $3 \mathrm{~m}$ & 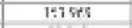 & $.7 \%$ & 134 & 1.18 \\
\hline Swi Sphastase & $8.4 t$ & Buts: & $\therefore$ in & 261 & i.i. \\
\hline Semili & $3 \times 143$ & $\sin x$ & $.4 \%$ & $\approx * 2$ & 1,45 \\
\hline Falare & $731 \mathrm{HS}$ & $4 \Gamma, 2 \pi$ & $.4,96$ & 5 & 17 \\
\hline Findedes & 202,48 & $11: 235$ & $4 \pi i$ & $2 a$ & $1, \alpha$ \\
\hline PHarts & 3.67 & 39:5: & $\sin x$ & 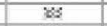 & $I N$ \\
\hline $2 \ln 1 \mathrm{ram}$ & $251 \% 3$ & Rinot & $2 \infty$ & 37: & $\omega 0$ \\
\hline WEEDLA & $2 n 2.0 n$ & $38=4 \mathrm{x}$ & $-41 \%$ & 154 & 1.24 \\
\hline TUKL & 45L1RE: & S.Lowe: & - & .. & - \\
\hline
\end{tabular}

Nota. Fuente: Población: INE, a 1 de enero de 2010, último censo nacional.

Datos oficiales: Fuente ayuntamientos y empresas contratadas en cada municipio.

(1) Toneladas de residuos.

La ley reguladora de los residuos en Cataluña, de 15 de julio de 1993, diferencia los residuos según el tipo de tratamiento que exige cada uno de ellos, clasificándolos en tres grupos:

a) Residuos especiales: Se consideran dentro de este grupo los disolventes orgánicos, éteres, compuestos organoclorados, plaguicidas, aceites industriales, baños de metales, cianuros, compuestos con metales pesados, y dioxinas.

b) Residuos no especiales: En este tipo se incluyen los embalajes de plástico, caucho, cueros, y textiles.

c) Residuos inertes: Es aquel residuo que, una vez depositado en un vertedero, no experimenta transformaciones físicas, químicas o biológicas significativas y cumple los criterios de lixiviación determinados por reglamento. Entre estos residuos tenemos: escombros de tejar, escorias, chatarra, y grava. 


\subsection{Residuos municipales, domésticos o residuos sólidos urbanos (RSU)}

La cantidad de residuos sólidos urbanos (RSU) generados depende de muchos parámetros: Nivel de vida de la población, época del año, movimientos de población (vacaciones, fiestas, fines de semana, etc.), métodos de envase y embalaje de productos.

- Bolsa de basura

Se va imponiendo el hecho de tener en casa dos recipientes, o solamente uno con dos compartimentos, para separar los restos corrientes. Uno de ellos para la materia orgánica (restos de comida, carnes, pescados, verduras, frutas, peladuras, restos de café...) que permitirá transformarla en compost y el otro para los materiales inertes más corrientes (productos inorgánicos, trozos de cristal, latas sucias, tazas rotas, tetrabriks, plásticos, restos metálicos...) que irán al vertedero o a una planta de selección.

\section{- Recolección selectiva}

Los contenedores adecuados para diversos materiales se encuentran en la calle, a ser posible cerca del ciudadano, en puntos de concentración de contenedores selectivos para papel-cartón, cristal, latas, plástico y tetrabriks. Otros residuos como pilas, medicamentos sobrantes o caducados, ropa, etc. se llevan a los lugares de recogida específica.

- Residuos domésticos que requieren una gestión especifica

Existen unos residuos que se han generado en el ámbito doméstico pero que por sus características requieren una gestión específica, siendo éstos: Colas y adhesivos, pinturas, barnices y disolventes, insecticidas y plaguicidas, aceites minerales de origen doméstico, aditivos y otros fluidos de automoción, residuos eléctricos y electrónicos, productos de la limpieza viaria y acumuladores fuera de uso.

\section{- Residuos comerciales}

Son los residuos municipales generados por la actividad propia del comercio, hostelería, bares, mercados, oficinas y servicios; residuos originados en la industria que tengan la consideración de asimilables a los municipales.

La gestión de los residuos comerciales corresponde al titular de la actividad que los genera y debe gestionarlos de acuerdo con las obligaciones propias de los productores de residuos.

Debe contactar con un gestor autorizado para su valorización, y si es posible, librarlos a un punto limpio o deposición en vertedero controlado.

La producción media en España de los distintos componentes de los residuos sólidos urbanos es la mostrada en la figura 3 (año 2007):

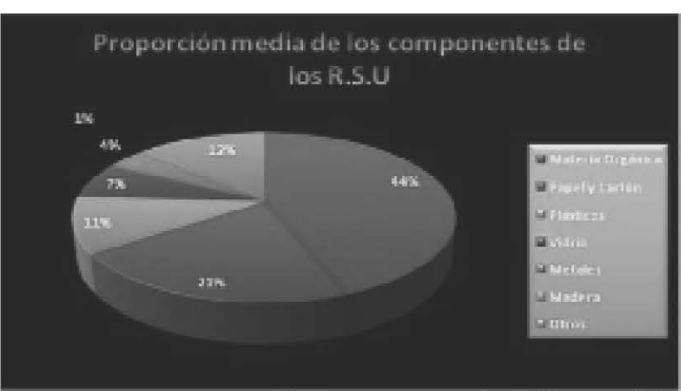

Figura 3. Porcentaje de los componentes de residuos sólidos durante el año 2007.

\subsection{Gestión de residuos sólidos urbanos}

La gestión de los residuos es el conjunto de actividades encaminadas a dar a los mismos el destino más adecuado y de acuerdo con sus características, para la protección de la salud humana, los recursos naturales y el medio ambiente. La gestión comprende la recogida, almacenamiento, transporte, valorización y eliminación de residuos, incluida la vigilancia de estas actividades, así como la vigilancia de los lugares de depósito o vertido después de su cierre.

Abarca tres etapas: Depósito y recogida, transporte y tratamiento

Sistemas de recolección de residuos:

- Vía pública, cercana al domicilio

Los contenedores son, desde la década de los ochenta, el sistema tradicional de recogida de basura doméstica y comercial que generan los ciudadanos; suelen localizarse cercanos a los domicilios, a distancias inferiores a los 50 metros.

La evolución urbanística experimentada por las ciudades y los cambios introducidos en el modelo de gestión de los residuos ha exigido, la incorporación de modificaciones en los modelos de contenedores que se han ido utilizando.

Se ha ido modernizando el diseño y optimizando el vaciado para adaptarlo a las características de las calles, a las necesidades de los ciudadanos y a los nuevos sistemas de recogida selectiva (Fig. 4).

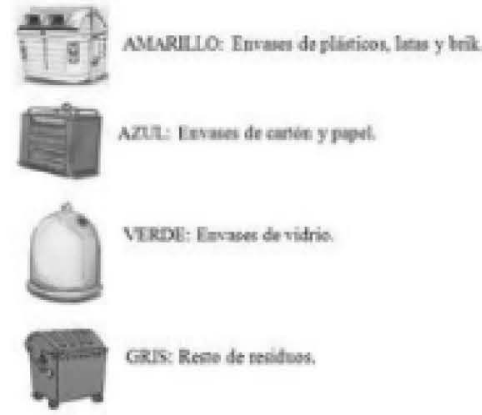

Figura 4. Tipos de contenedores según la tipología residual.

En zonas de nueva construcción o en zonas ya edificadas, en las que los condicionantes urbanísticos y económicos lo permiten, es posible soterrar los contenedores.

\section{- Carga lateral}

Esta denominación hace referencia a los vehículos de recogida adaptados, que recogen de forma automatizada 
los contenedores: El camión se coloca junto al contenedor, lo eleva y lo vacía con la ayuda de unos brazos articulados. Los contenedores de carga lateral se usan para recoger diferentes fracciones de residuos (figura 5). La fracción recogida viene determinada por el color del contenedor. Su capacidad es de 3.200 litros, lo que supone una disminución del número total de contenedores en la vía pública, y un incremento de la relación volumen/superficie.

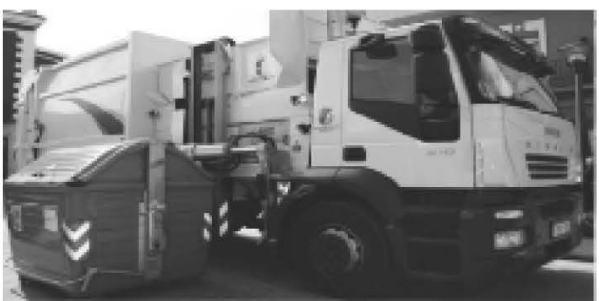

Figura 5. Contenedor de carga lateral.

\section{Recolección bicompartimentada}

El presente modelo consiste en contenedores que recogen de forma segregada la materia orgánica y los materiales de rechazo no reciclables, que no pueden ser depositados en ningún otro contenedor de recogida selectiva o llevados a los centros de recuperación y reciclaje (papel y plásticos sucios, papel de aluminio, restos de cerámica, artículos de piel, lana, algodón, etc.). Cada compartimento está identifícado mediante un color diferente, lo que facilita la separación en origen de la basura.

La principal ventaja es la reducción del número de viajes que tienen que realizar los camiones de recogida, ya que, al tratarse de vehículos adaptados, pueden retirar a la vez ambas fracciones.

Esta modalidad se ha implantado, hasta el momento, de forma pionera, en diferentes zonas de la ciudad de Barcelona, Hospitalet de Llobregat y Ripollet.

- Recolección simultánea en bolsas de color

Existen municipios que han optado por emplear el contenedor habitual para la recogida de la fracción orgánica de forma simultánea a la de la fracción resto. Con la finalidad de diferenciar las dos fracciones, los ciudadanos tienen que depositar los residuos orgánicos en bolsas de color naranja y el resto en bolsas convencionales. Las bolsas con diferentes fracciones son recogidas por un único camión y separadas según su color mediante un sistema de selección óptica Optibag, que consiste en un sistema de lectores ópticos que distingue las bolsas por su color. Badalona y Barcelona son los municipios que han puesto en práctica este sistema de recogida y el Ecoparque de Barcelona (figura 6) cuenta con este sistema de separación.

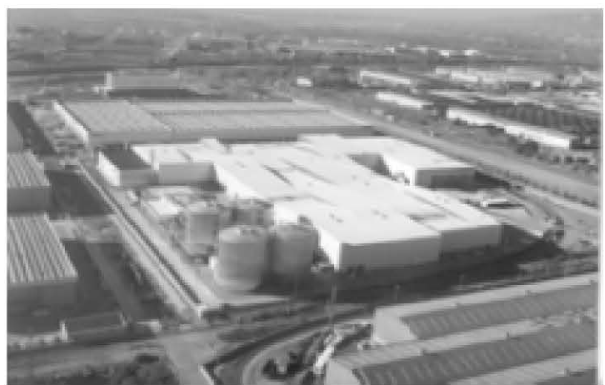

Figura 6. Ecoparq 1 general 3 (Barcelona).

\section{- Contenedores soterrados}

Estos permiten almacenar los residuos bajo tierra, lo que supone una serie de ventajas: Ganar espacio a la calle, reducir el impacto visual, y el ruido así como disponer de un entorno más limpio.

Éste es el sistema por el que ha optado el municipio de Castellbisbal y el aplicado en Barcelona en varias zonas de la ciudad (Rambla de Prat, plaza Santa Madrona, plaza Vila de Madrid, etc.).

- Áreas de aportación

Son aquellas zonas situadas a un máximo de 150 metros de distancia de los domicilios. Normalmente se recomienda que se agrupen en un ratio de 500 habitantes/contenedor.

- Puerta a puerta

Se recogen las diferentes fracciones de residuos domésticos en la puerta de las viviendas en unos días determinados de la semana.

En enero de 2003, Torrelles de Llobregat también estrenó esta modalidad de recogida puerta a puerta. Todos los contenedores, salvo el del vidrio, han sido retirados de la vía pública y la basura tiene que dejarse en la puerta de las viviendas en una franja horaria determinada. La materia orgánica, inorgánica y papel se recogen tres, dos y un día por semana, respectivamente.

- Sistemas neumáticos

Los residuos son transportados a través de cañerías subterráneas desde el lugar en el que se producen (viviendas, locales comerciales, etc.) hasta una central de recogida o un camión específico. La basura se deposita en unos buzones instalados en el interior de los edificios -en aquellos que ya incorporan este sistema-, o en la vía pública, y se compacta en contenedores herméticamente cerrados antes de ser transportada al lugar en el que se procede a su tratamiento final. Hasta ahora, el sistema ha sido implantado en diferentes zonas de los municipios de Barcelona, Badalona y Cerdanyola del Vallès.

Es necesario distinguir dos tipos:

a) Sistema fijo (figura 7): El ciclo de recogida se inicia en los rellanos de las viviendas o en la calle. Las bolsas son depositadas a través de una compuerta en un tubo vertical que hace las veces de bajante. La basura cae por efecto de la gravedad hasta la válvula que está instalada en el sótano, y que se mantiene cerrada, donde es almacenada. La válvula de funcionamiento neumático conecta con una red de cañerías horizontales que llegan a la central de recogida.

b) Sistema móvil (figura 8a): El ciclo de recogida se inicia en la calle, donde se han instalado una especie de buzones de correo (figura 8b), conectados entre sí por 
debajo del suelo. La aspiración se realiza desde un camión que se conecta a uno de los extremos del circuito.

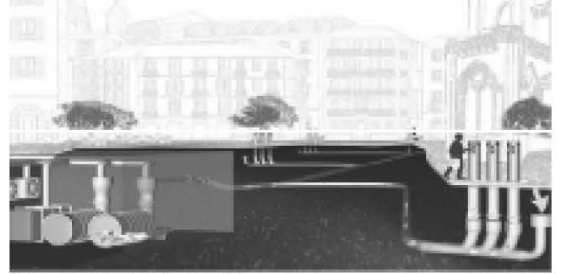

Figura 7. Sistema fijo. a)

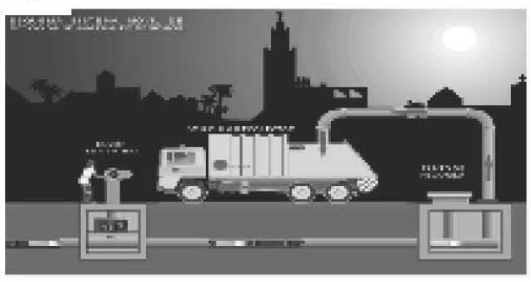

b)

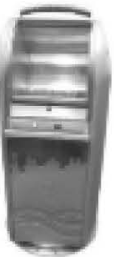

Figura 8. a) Sistema móvil y b) Buzón de basura.

En una investigación llevada a cabo en el año 2012, se detallan el número de contenedores y de puntos limpios por habitante en varias provincias españolas (Tabla 2).

Tabla 2. Recogida selectiva: Número de contenedores y de puntos limpios por habitante en el año 2011.

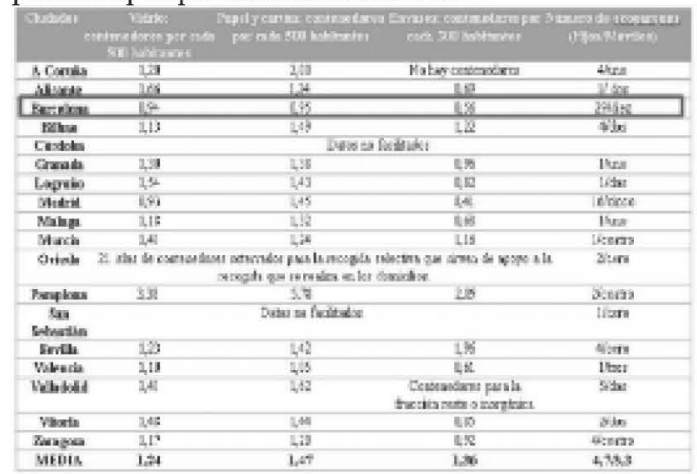

Nota. Fuente: Ayuntamiento de cada ciudad o, en su caso, la empresa encargada de la recogida selectiva de los residuos.

Transporte de residuos.

Esta fase comprende una serie de operaciones que son necesarias para la retirada de los residuos sólidos urbanos (figura 9), desde el punto en que los abandona el productor hasta su descarga en el lugar de eliminación o instalación de aprovechamiento, o bien pasar por una fase intermedia o estación de transferencia. Esta última operación se realiza cuando el lugar de recogida y el punto de destino se encuentran a una distancia superior a $15-20 \mathrm{~km}$.

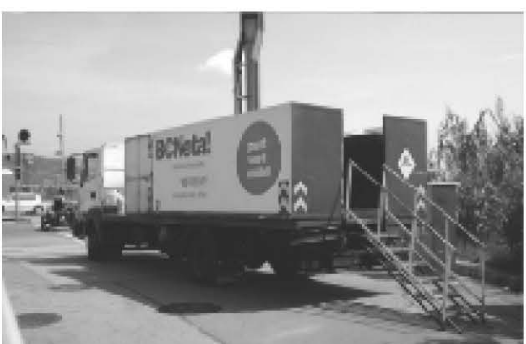

Figura 9. Transporte de Residuos.

Tratamiento de los residuos sólidos urbanos.

Constituye la fase final del proceso, siendo preciso someter la basura a una selección previa antes de tratar los residuos aunque haya existido un sistema de recogida selectiva. El citado proceso consiste en separar la materia orgánica de otros materiales que se encuentran mezclados con aquella.

En lo que respecta al tipo de instalaciones de tratamiento de residuos urbanos, se pueden distinguir las siguientes:

a. Estación de transferencia (figura 10).

Es un depósito transitorio que cumple la función de comprimir la basura para transportarla posteriormente a una planta de tratamiento. Posee una gran superficie donde se reciben los distintos residuos, se clasifican en función de su tipología, se almacenan hasta tener una cantidad suficiente y se derivan hasta un gestor final que los trata y reincorpora a la cadena de producción.

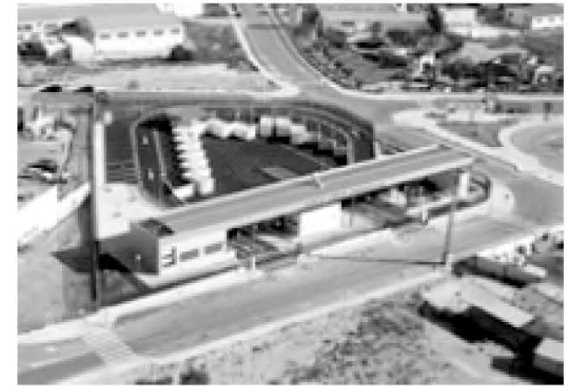

Figura 10. Planta de transferencia.

\section{b. Planta de compostaje (figura 11).}

Se trata de un centro productivo donde se clasifica la basura con objeto de transformar la materia orgánica en compost. Con el compostaje se produce la degradación biológica de los polímeros lignocelulósicos, transformándolos en humus, mezcla de ácidos húmicos y fúlvicos, que es la materia orgánica idónea para el suelo.

El compostaje es un proceso de fermentación aeróbica de carácter exotérmico, que es llevado a cabo por los microorganismos, produciéndose en dos etapas; una mesófila de carácter degradativo y otra de maduración, donde se fuerzan y ultiman los procesos oxidantes y se estabiliza el producto. Además el compuesto devuelve al suelo buena parte de los nutrientes - nitrógeno, fósforo y potasio-, contenidos en los restos.

La materia orgánica recogida selectivamente y los restos de poda y jardinería que se recogen en el área metropolitana pasan por una línea de tratamiento para obtener compost. A través de dos plantas se tratan anualmente 10.000 toneladas de materia orgánica. 


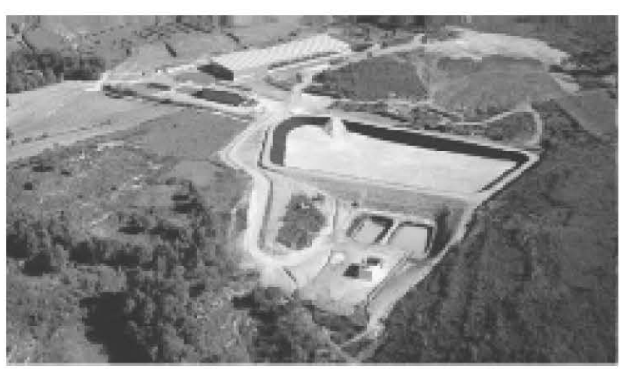

Figura 11. Planta de compostaje. Espluga de Francolí (Tarragona).

La planta industrial de compostaje, se encarga de reciclar la fracción de materia orgánica contenida en la basura (algo más del 40\%) y posibilita su aprovechamiento como abono en la agricultura. El producto obtenido, denominado compost, supone algo más de la mitad de la materia orgánica tratada, ya que el resto es fundamentalmente agua que se evapora en los procesos de secado y fermentación.

Además de la citada planta, este complejo incluye instalaciones de recuperación selectiva de papel, plásticos, vidrio, materiales férricos, y subproductos cuya fracción aprovechable alcanza el $8 \%$ del residuo urbano total. La fracción resto, compuesta por materiales de rechazo no reutilizables, se destina al vertedero controlado (figura 12).

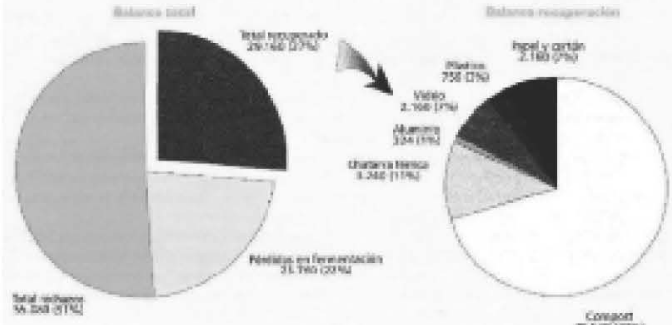

Figura 12. Producción de compost y balance de recuperación

\section{c. Planta de biometanización}

Es un tipo de instalación en la que se produce biogás (figura 13) mediante un proceso de degradación anaeróbica de la materia orgánica. Posteriormente, la fracción sólida resultante es tratada para obtener compost.

Se incentiva la generación de corriente eléctrica tendiéndose a producir biogás a partir de desechos orgánicos de todo tipo: Excrementos animales, restos de cultivo agrícola, estiércol, alimentos en mal estado, productos de industrias agroalimentarias, etc. (Tabla 3 ). Una fermentación anaeróbica en grandes tanques genera al cabo de un tiempo variable (15 días a 2 meses), un biogás con un $65 \%$ de metano y $30 \%$ de $\mathrm{CO}_{2}$ y un poder calorífico superior a $5.000 \mathrm{kcal} / \mathrm{kg}$. Se conduce el biogás a un gasómetro y de éste a una central de cogeneración con motores de generación eléctrica para entregar energía a la red.

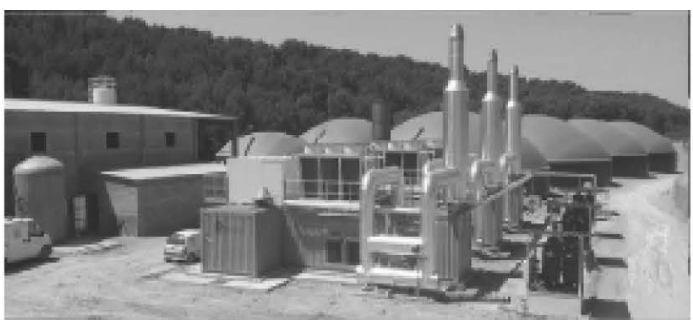

Figura 13. Planta de biogás de Almenar, Segrià.

Tabla 3. Tabla de producción de biogás, según la materia orgánica utilizada (9 de Enero de 2015).

\begin{tabular}{|c|c|c|}
\hline Notnangers & 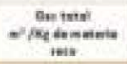 & 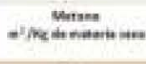 \\
\hline 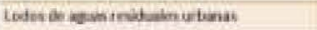 & a, 43 & $0=4$ \\
\hline Rasumiphenen & Satit & ase \\
\hline 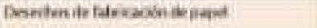 & e.21 & 10.14 \\
\hline 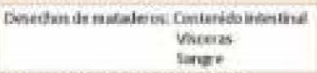 & $\begin{array}{l}0,47 \\
\text { s.in } \\
\text { a.16 }\end{array}$ & $\begin{array}{l}0.35 \\
\text { inis } \\
\text { oin }\end{array}$ \\
\hline 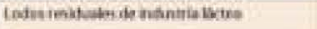 & wa & 0.4 \\
\hline lindos resaluke de papriersi & 0.25 & Q13 \\
\hline 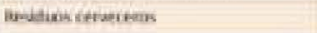 & 041 & Q11 \\
\hline 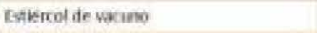 & 0,4 & 099 \\
\hline Estierni de postim & 0.26 & क्य \\
\hline Nebinde piatian. & 0,51 & 0,40 \\
\hline Hoglas the main & 0,49 & 0,41 \\
\hline Hogn de remibath & 0.46 & axs \\
\hline
\end{tabular}

Una investigación llevada a cabo en el año 2010 , muestra que el biogás podría suplir el $12 \%$ del consumo de gas natural; el biogás se convierte, en un $99 \%$, en electricidad. España se sitúa en una posición privilegiada en materia de biogás (figura 14) siendo el motivo la intensa actividad agroalimentaria en nuestro país, que genera unos residuos que son potencialmente reconvertibles en un gas limpio y sin emisiones de gases de efecto invernadero.

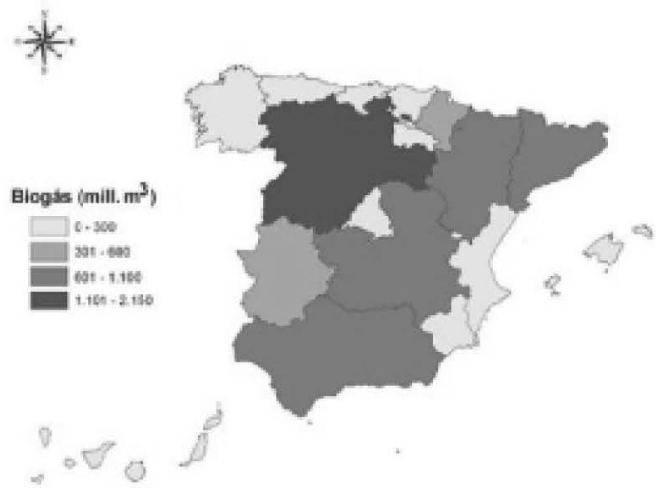

Figura 14. Mapa del potencial de biogás en España.

d. Planta de valorización energética (figura 15).

Los residuos son sometidos a altas temperaturas, obteniéndose gases. La energía calorífica de éstos, es aprovechada para su transformación en electricidad. 


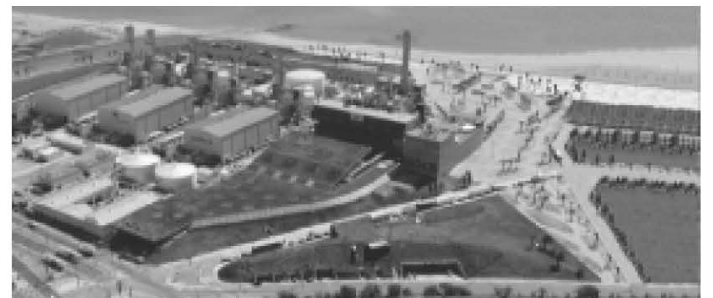

Figura 15. Planta Integral de Valorización de Residuos (PIVR) de Sant Adrià de Besòs; se trata de una instalación de tratamiento integral de los residuos municipales del área Metropolitana de Barcelona.

\section{e. Incineradora.}

Es una instalación que dispone de una caldera de combustión donde se quema la basura a una elevada temperatura (figura 16). El vapor generado, se aprovecha para alimentar una turbina que produce electricidad.

Los residuos previamente autorizados, llegan a la planta en camiones de caja cerrada y compactados, que se vierten a la fosa de almacenaje. Una grúa recoge los residuos y los introduce en el horno, donde pasan a la cámara de combustión y de post-combustión.

Los gases que se producen, pasan a la caldera de recuperación, donde se genera el vapor recalentado que, mediante un turboalternador, se convertirá en energía eléctrica. Los excedentes pasan a una planta, a los filtros y al lavado de gases antes de salir por la chimenea.

Los gases pasan primero por una planta donde se separan las partículas sólidas que contienen (cenizas), que unidas a las de la caldera reciben un tratamiento como residuos especiales. La parte de gases sucios se mezclan con lechada de cal pulverizada, que provoca una reacción química de neutralización. Posteriormente, se separan las partículas absorbidas y los gases limpios se liberan a la atmósfera por una chimenea de unos 50 metros de altura.

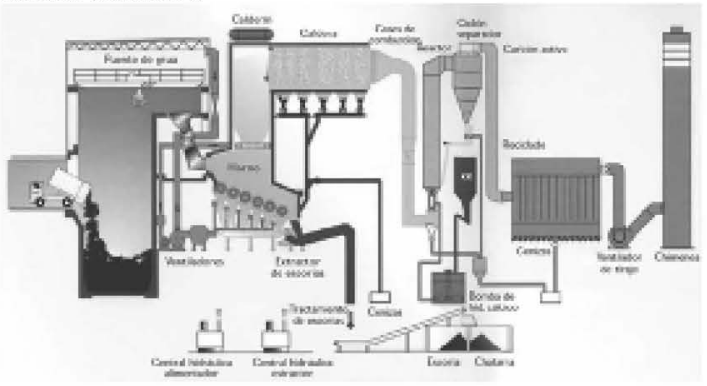

Figura 16. Incineradora de residuos municipales.

\section{f. Vertedero controlado (relleno sanitario).}

Es un espacio reservado para depositar los residuos compactados y no aprovechables, después de haber sido sometidos a un tratamiento; los residuos interaccionan entre ellos y con el agua de lluvia, en procesos que pueden durar desde segundos hasta decenas de años, y se producen compuestos mineralizados (sólidos), lixiviados (líquidos) y biogás (gases).

Los depósitos controlados de mayor seguridad requieren de una impermeabilización por debajo de las capas de residuos, y de unas zonas drenantes de arcilla y tierra vegetal por encima (figura 17); cuando llega al final de su vida útil, deben clausurarse.
Los lixiviados proceden de la reacción y disolución de los componentes solubles de los residuos por el agua de aportación o por la humedad que ellos mismos contienen.

Para garantizar que estos lixiviados no lleguen a las aguas subterráneas, con la consecuente contaminación, es necesario impermeabilizar el terreno donde se ubica el depósito controlado y disponer de un sistema de recolección de los líquidos, con un drenaje de gravas o arenas, para su posterior tratamiento.

Las arcillas son un buen material impermeabilizante, que por su composición química, actúan como filtro de retención de los metales pesados.

Debido a la degradación de la materia orgánica se producen gases como el nitrógeno $\left(\mathrm{N}_{2}\right)$, dióxido de carbono $\left(\mathrm{CO}_{2}\right)$, metano $\left(\mathrm{CH}_{4}\right)$ y en menor cantidad monóxido de carbono (CO) y otros. En general, se dispone de un sistema de drenaje de gases mediante tubos metálicos llenos de grava o materiales similares.

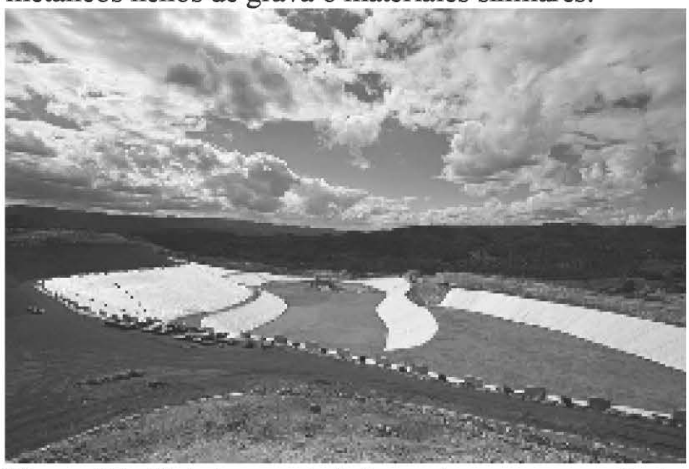

Figura 17. Vertedero controlado en el que una capa de dos metros de aislantes, separa los residuos del suelo.

Además de los centros de tratamiento citados, también se distinguen otro tipo de instalaciones, denominadas puntos limpios o ecoparques (figura 18), que son recintos cerrados donde los ciudadanos pueden llevar determinados tipos de residuos (muebles, electrodomésticos, pilas, aceites, televisores, tubos fluorescentes o bombillas) que por su volumen o toxicidad no son recogidos en los contenedores de basura orgánica.

Todos estos residuos se almacenan de forma temporal para su transporte a plantas de selección, donde se separan, recuperan y reciclan. Las fracciones no aprovechables se llevan a vertederos controlados.

La función principal del ecoparque es valorizar la fracción orgánica y el resto (residuos que no se pueden recoger selectivamente pero contienen materiales recuperables) a través de diversos tratamientos complementarios como la selección y separación de materiales valorizables, el compostaje y la metanización.

La materia orgánica procedente de la recolección selectiva domiciliaria, de comercios, mercados y restos de jardinería se destina al compostaje de calidad, con etapas de fermentación y maduración.

De la fracción resto se recuperan los materiales reciclables, como papel, vidrio, plástico y envases, utilizando sistemas de triaje: Tambores rotativos, electroimanes y separadores de inducción para los envases, además de la selección manual.

La fracción orgánica restante de poca calidad, no compostable, se puede metanizar para obtener biogás. E1 
metano se transforma en energía eléctrica por combustión.

El desecho final no reciclable ni recuperable, procedente de la fracción resto, se puede inertizar, compactar y verter a un depósito controlado, sin ningún efecto negativo para el medio.

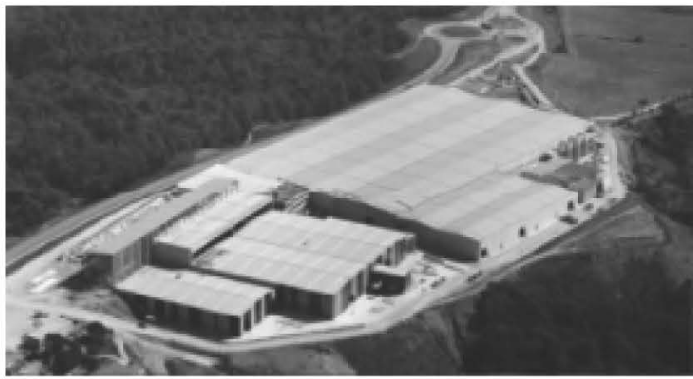

Figura 18. Ecoparc 4, ubicado en Els Hostalets de Pierola (Barcelona) para tratar anualmente unas 400.000 toneladas de la fracción resto.

España cuenta con 1.238 puntos limpios, bastante repartidos por el país, dando servicio a la mayoría de la población, y en la figura 19 se muestra la ubicación de la red de puntos limpios del Área Metropolitana de Barcelona.

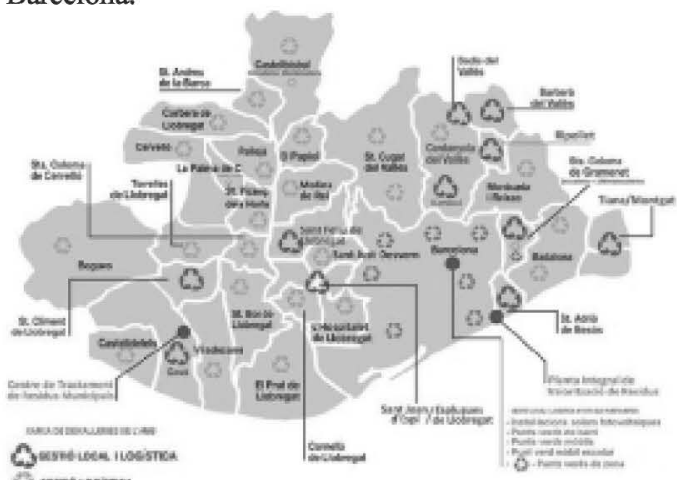

Figura 19. Ubicación de la red de puntos limpios del Area Metropolitana de Barcelona

Un análisis presentado a principios del año 2015 , refleja el número de personas que utilizan la red metropolitana de Barcelona de puntos limpios (figura 20) $\mathrm{y}$ el número de toneladas que recibe la citada red (figura 21) desde el año 2010 a 2014.

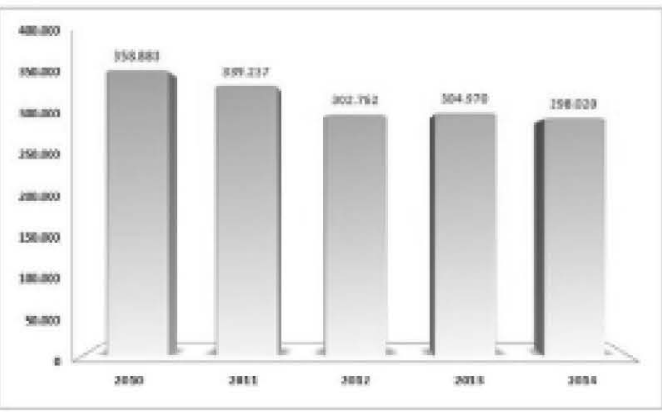

Figura 20. Usuarios de la red metropolitana de puntos limpios (TERSA, 2015)

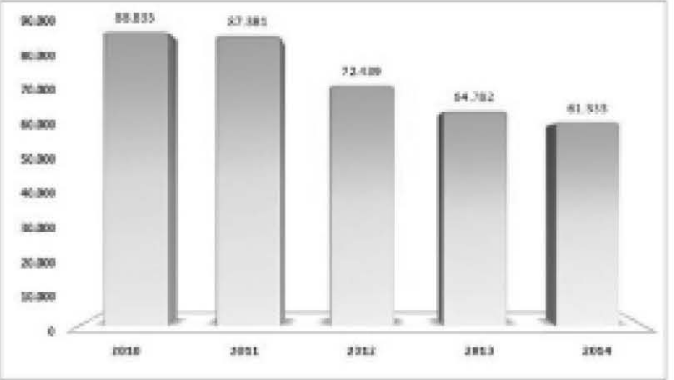

Figura 21. Toneladas de la red metropolitana de puntos limpios (TERSA, 2015).

\subsection{Legislación de residuos sólidos urbanos}

La legislación en materia de residuos en España se debe, en parte, a las transposiciones de las Directivas Europeas.

La Comunidad Autónoma de Cataluña dispone de competencias en materia de residuos, y por tanto existe una legislación aplicable en todo el territorio autonómico.

Es necesario destacar tres leyes fundamentales, de aplicación en todo el estado español:

- La Ley 7/1985 de Bases de Régimen Local que establece la recogida de residuos como servicio obligatorio para los municipios, sea cuál sea el tamaño del municipio. Los municipios de más de 5.000 habitantes deben garantizar también el tratamiento de los residuos.

- La Ley 10/1998, de 21 de abril, de residuos, establece las orientaciones básicas y las obligaciones para los municipios y productores. Actualmente, en España es obligatoria la recogida selectiva de los residuos. De esta ley se deriva el Plan Nacional de Residuos (2000-2006), aprobado el 7 de enero del 2000.

- Ley 11/1997, de 24 de abril, de Envases y Residuos de Envases, establece que los envases son propiedad de los productores o de los envasadores y que los usuarios deben devolverlos a través de los comercios mediante un sistema de depósito o bien a través de sistemas integrados de gestión.

En los Sistemas Integrados, el municipio se encarga de mantener contenedores en la vía pública, recoger su contenido y trasladarlo hasta las plantas de reciclaje. La asociación de productores debe hacerse cargo del sobrecoste de operación y aportar recursos económicos al municipio.

En la Comunidad Autónoma de Cataluña el papel de la administración municipal se refuerza a través de la Ley 8/1987 Municipal y de Régimen Local de Cataluña que establece la recogida de residuos como servicio obligatorio para los municipios.

Además:

- Como normativa general, es necesario considerar la Ley $6 / 1993$, reguladora de residuos, modificada por la ley 15/2003. En esta reglamentación básica se establece la separación entre los residuos municipales y los residuos generados por los comercios y actividades económicas. Cada comercio puede optar por contratar un servicio privado de recogida de residuos o satisfacer el importe de la gestión al servicio municipal. Se establece también 
la obligación de los municipios de organizar la recogida selectiva de residuos orgánicos y de mantener una deixalleria (punto limpio) en la que los ciudadanos pueden aportar residuos peligrosos o voluminosos.

- Decreto 92/1999 que aprueba el Catálogo de Residuos. - Decreto 217/1999 que regula la gestión de los vehículos fuera de uso o abandonados.

- Ley 9/2000, de regulación de la publicidad dinámica a Cataluña. La normativa trata de evitar la generación de residuos de la publicidad indiscriminada sobre papel que se reparte en los domicilios.

- Ley 11/2000, reguladora de la incineración de residuos. - Ley 16/2003, de financiación de las infraestructuras y del canon. Esta norma, que ha empezado a aplicarse el año 2004, establece un canon de 10 euros por tonelada de residuo que se disponga en depósito controlado. Este canon se añade al precio de deposición y sirve para financiar las infraestructuras de tratamiento no finalista como las plantas de compostaje o los ecoparques que utilizan tecnologías de compostaje y metanización.

Cada municipio puede organizar el servicio de recogida de acuerdo con la normativa general vigente. Normalmente, cada ayuntamiento redacta y aprueba unas ordenanzas ambientales que establecen, como mínimo, como será el servicio de limpieza de las calles y de recogida de basura. Se establecen las fracciones y los horarios de recogida de la basura, el tipo de contenedores o bolsas que deben utilizarse para entregar los residuos a los gestores autorizados.

La ciudad de Barcelona y los 32 municipios que la rodean optaron por gestionar de forma conjunta sus residuos. La Entitat Metropolitana de Serveis Hidràulics i Tractament de Residus es el ente creado con la finalidad de gestionar las instalaciones de tratamiento necesarias para aplicar el Programa Metropolitano de Gestión de Residuos Municipales. Este Programa, con un periodo de aplicación de 1997 a 2006, establece las fracciones en las que se deben separar los residuos y las plantas de tratamiento necesarias. En el año 2004, se han dispuesto de 40 deixalleries, 2 ecoparques que incorporan plantas de compostaje y metanización, 3 plantas de compostaje, 2 incineradoras, 1 planta de selección de envases y residuos de envases y un depósito controlado en proceso de clausura.

\subsection{Educación ambiental}

En los recientes acuerdos internacionales, Agenda 21, en la Conferencia de las Naciones Unidas sobre Medio Ambiente y Desarrollo (Río de Janeiro, 1992) marcó como objetivos en el ámbito de los residuos, un cambio en las pautas de producción y consumo de manera que se minimicen los residuos que se generan y se incremente la minimización y el reciclaje. Se aboga por dejar atrás la actual cultura de "usar y tirar" y considerar que el residuo no es un problema por sí mismo, sino que éste es el resultado de determinados hábitos de consumo y de nuestro comportamiento ciudadano y humano.

Es necesario educar, y sensibilizar, para incidir y potenciar los comportamientos ecológicamente responsables. Hay que desarrollar efectivos programas educativos tanto en centros escolares, como los destinados a toda la población, de manera constante, intentando abarcar especialmente a aquellos sectores de la población, más directamente relacionados con la producción y la gestión de los residuos.

Solo lograremos éxito con la finalidad de involucrar activamente al ciudadano si somos capaces, de abrir a la participación los procesos de gestión, de estudio, y sobre todo, de toma de decisiones.

La educación ambiental es un proceso multidimensional e integral de adquisición de saberes (conocimientos), destrezas (para saber hacer: experiencias, competencias) y valores (para "saber estar") que al combinarse permiten responder activamente a la crisis ambiental.

La formación ambiental define las enseñanzas que toman como referencia un campo específico y especializado de las ciencias o saberes sobre el medio ambiente [1].

En Cataluña, se están impulsando programas educativos en algunos municipios, "Red de Escuelas Verdes", promoviendo una gestión sostenible integral de los centros.

Otras experiencias están basadas en la construcción y dinamización de centros especializados, como Centros de Educación Ambiental, Centros de Interpretación de la Naturaleza, Casas de Naturaleza, o Centros de Divulgación de la Gestión de la Basura.

Son muchas también las experiencias basadas en una multitud de publicaciones informativas y divulgativas sobre cuestiones ambientales y el amplio mosaico de la gestión de los residuos; cabe mencionar Compartamos un Futuro y Campaña Puerta a Puerta.

Las campañas temporales, en los distintos soportes audiovisuales, impresos, TV, etc., y especialmente las visitas colectivas a los centros de tratamiento de los residuos acostumbran a dar buenos resultados.

La información y la comunicación pretenden hacer llegar a la ciudadanía informaciones o pautas instrumentales para el mejor desarrollo de sus responsabilidades; entre las actividades de que se pueden llevar a cabo se encuentran: Campañas específicas de concienciación, páginas web con espacio de consultas interactivo, revistas o boletines locales, programas de radio y televisión, carteles, exposiciones de materiales o productos reciclados, visitas a las instalaciones de gestión de residuos, talleres de compostaje en casa o de papel reciclado.

Una participación efectiva de la ciudadanía puede conseguir diversos objetivos:

1) Educar, es decir, ayudar a formar una opinión, cambiar actitudes y modificar comportamientos.

2) Acceder a una información más detallada y fiable que facilite la toma de decisiones más informadas.

3) Legitimar determinadas políticas.

4) Garantizar la corresponsabilidad en la gestión de los intereses generales.

El Area Metropolitana de Barcelona para hacer el seguimiento del Programa de Residuos se ha dotado de un organismo participativo, que integra a todos los sectores sociales y agentes activos. Se trata del Consejo de Seguimiento del Programa Metropolitano de Gestión de Residuos Municipales.

Este Consejo está formado por representantes políticos, representantes de todas las universidades catalanas, sindicatos, organizaciones empresariales, 
grupos ecologistas, asociaciones vecinales y también expertos en la gestión de residuos.

La finalidad básica de este Consejo, es hacer el seguimiento de la evolución e implantación del Programa Metropolitano, estudiar, debatir sus elementos teóricos, proponer alternativas, asesorar al gobierno en este campo y ayudar a comunicar mejor sus planteamientos.

El Consejo dispone de tres comisiones de trabajo para estudiar los temas más específicos. Estas comisiones están presididas por representantes de la Universidad, y son la Comisión de Financiación, que estudia los aspectos relacionados con la economía del programa, ingresos, tasas, etc.; la Comisión de Infraestructuras, encargada de estudiar las propuestas de instalaciones, plantas, tecnologías, etc.; y la Comisión de Despliegue, que se ocupa del avance y desarrollo general del programa, y de su implantación en los municipios.

Estas comisiones elaboran propuestas y documentos, que luego son aprobados por el Consejo de Seguimiento y elevadas a los órganos políticos pertinentes, el consejo de administración de la Agencia Metropolitana de Residuos o el Consejo Plenario de la Entidad Metropolitana del Medio Ambiente.

\section{Resultados}

Actualmente, está aumentando la producción de residuos debido a un crecimiento de la población mundial, concentración de la población en núcleos urbanos, utilización de envases en muchos productos, uso generalizado de los envases sin retorno, y temprana obsolescencia de los artículos (figura 22). Asimismo, también se representa la evolución de la recogida selectiva en la comunidad catalana desde el año 2001 hasta el año 2008 (figura 23), reflejándose que en el año 2010 en la provincia de Cataluña se recogieron selectivamente 1.701 .100 toneladas de residuos; el índice de recogida selectiva se sitúa en el $40,57 \%$ del total de residuos generados y la tendencia de la recogida selectiva de residuos continúa al alza.

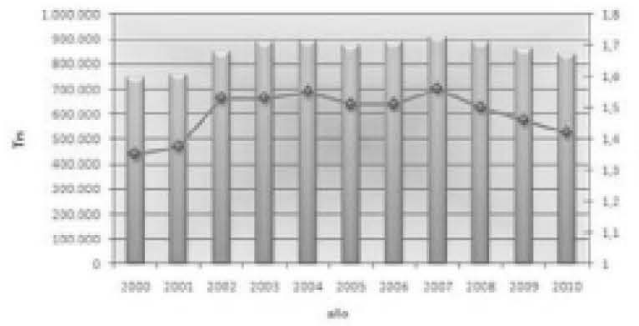

Figura 22. Evolución de la generación de residuos municipales en el Area Metropolitana de Barcelona (Dades Ambientals Metropolitanes, 2010).

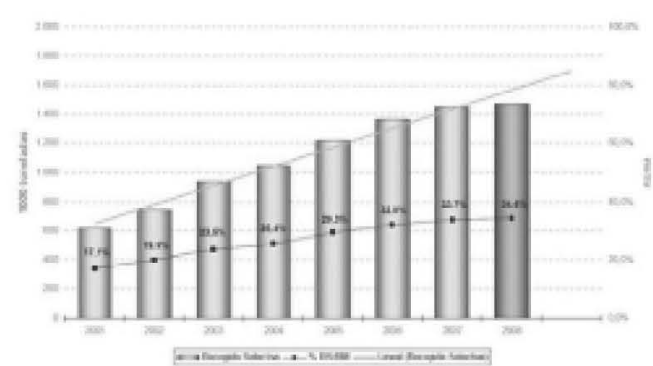

Figura 23. Evolución de recogida selectiva de residuos municipales en el Area Metropolitana de Barcelona.

Adicionalmente, una mala gestión de los residuos da lugar a: Contaminación del suelo, acuíferos, emisión de metano de los vertederos (explosiones e incendios), impacto visual y contaminación atmosférica (cenizas y dioxinas).

Una de las mejores soluciones para cuidar el medio ambiente, es una propuesta sobre hábitos de consumo popularizada por la organización ecologista Greenpeace. Este concepto hace referencia a estrategias para el manejo de residuos que buscan ser más sustentables con el medioambiente y específicamente dar prioridad a la reducción en el volumen de residuos generados; todo ello está enfocado a aplicar la regla de las $3 \mathrm{R}$ (reducir, reutilizar y reciclar):

- Reducir: Consiste en minimizar la cantidad de residuos producidos; se trata de disminuir o eliminar la cantidad de materiales destinados a un uso único, adaptar los aparatos en función de sus necesidades, moderar pérdidas energéticas o de recursos

- Reutilizar: Corresponde a la acción de volver a utilizar los bienes o productos, y darles otro uso.

- Reciclar: Es un proceso por el cual recuperamos total o parcialmente materia prima reutilizable de un producto ya elaborado.

Un análisis elaborado en el año 2012, indica que la tasa de reciclaje en España de papel se situó en el 73,5\%, una de las más altas del mundo (figura 24).

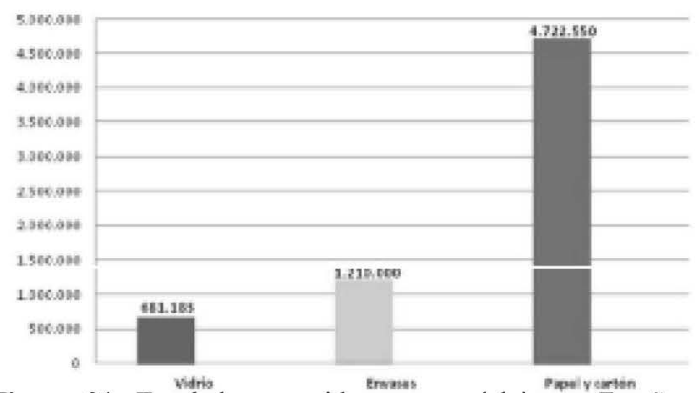

Figura 24. Toneladas recogidas para reciclaje en España (Ecovidrio, Ecoembes y Aspapel, 2012).

\section{Conclusiones}

Para una correcta gestión de los residuos urbanos, las diferentes Administraciones deberán concienciar a la sociedad del valor potencial que encierran los residuos que ésta genera, y la necesidad de su reutilización. No sólo hay que tener en cuenta la riqueza que suponen los distintos materiales desechados, sino la serie de 
beneficios indirectos que este tipo de gestión implica: Menor ocupación del suelo destinado al vertido, ahorro energético $\mathrm{y}$, fundamentalmente, disminución de la contaminación.

Dado que con el reciclaje de los residuos se consigue recuperar una parte de los materiales, debe potenciarse la recogida selectiva y más aún, la separación en origen. Hacia estas dos últimas formas de gestión deberán ir encaminados los futuros planes de gestión de los Residuos Sólidos Urbanos.

Por otra parte, la Educación Ambiental debe ser un proceso permanente en el que los individuos y la colectividad cobren coherencia con su entorno y adquieran los conocimientos, competencias, experiencias y la voluntad capaces de hacerlos actuar individual y colectivamente para resolver los problemas actuales y futuros del Medio Ambiente.

\section{Referencias}

1. Asociación Mundial de las Grandes Metrópolis http://www.metropolis.org (accesado el 2 de febrero de 2015).

2. Ecovitrum. http://www.ecovitrum.eu (accesado el 16 de febrero de 2015)

3. Escola Universitaria de Enxeñería Técnica Industrial. http://www.eueti.uvigo.es (accesado el 23 de enero de 2015).

4. Fundación

Cajamar. http://www.publicacionescajamar.es (accesado el 9 de febrero de 2015).

5. Sauvé, L. Exploración de la diversidad de conceptos y de prácticas en la educación relativa al ambiente. En $L a$ Dimensión Ambiental y la Escuela. Bogotá, 1994.

6. Sustentator. http://sustentator.com (accesado el 3 de febrero de 2015)

7. Tecnum. Escuela de Ingenieros. Universidad de Navarra. http://www.tecnun.es (accesado el 28 de enero de 2015).

8. Mitchell, B. La Gestión de los Recursos y del Medio Ambiente. Ediciones Mundi-Prensa. Barcelona, España, 1999.

9. Boletín de Medio Ambiente 2011: Departamento de Territorio y Sostenibilidad; Generalitat de Catalunya: España, 2012.

10. Gestión Integral de Residuos Sólidos; Tchobanoglous, G., Theisen A. and Vigil S.; McGraw Hill: Madrid, 1994

11. Bonmatí, A. Gestión y tratamiento de residuos sólidos urbanos. In Evaluación y prevención de riesgos Ambientales en Centroamérica; Andrés, P. y Rodríguez, R., Eds; Documenta Universitaria: Girona (España), 2008; pp. 215-250

12. Plan nacional de Residuos Urbanos (2000-2006); Ministerio de Medio Ambiente, Consejo de Ministros de España: Madrid, (2000).

13. Recursos; Departamento de Territorio y Sostenibilidad, Servicio de Educación Ambiental: Generalidad de Cataluña, 1999.

14. Compostatge i gestió de residus orgànics. Estudis i monografies 21; Soliva, M.; Diputació de Barcelona, Àrea de Medi Ambient: Barcelona, 2001.

15. Cavero, M.A.; Valorización energética de los residuos. Presentado en VII Jornadas técnicas de medio ambiente, Barcelona, Noviembre 16 - 17, 2011.

16. Ortega Rodríguez, M. Energías Renovables. Editorial Paraninfo. Madrid, España, 2003.

17. Legislación; Ministerio de Agricultura, Alimentación y Medio Ambiente: Madrid.

18. Libro blanco de la educación ambiental en España; Ministerio de Medio Ambiente: Madrid, 1999.
19. Societat Catalana D Educacio Ambiental L'ambientalització de la universitat, Núm.6.; Barcelona 1997.

20. Greenpeace. La situación de las basuras en España; Madrid, 2006. 